\title{
Some fixed point results via $\gamma$-contraction in non-Archimedean fuzzy metric spaces
}

\author{
Müzeyyen SANGURLU SEZEN* \\ Gazi University, Faculty of Science, Beşevler, 065000, Ankara, Turkey \\ Highlights \\ - The aim of this study is to obtain new results regarding the fixed point. \\ - Reich type $\gamma$-contraction and Kannan type $\gamma$-contraction are introduced. \\ - Some new results are proved in non-Archimedean spaces.
}

\section{Article Info}

Received: 19 Nov 2020

Accepted: 28 July 2021

\section{Keywords}

Reich type $\gamma$-contraction Kannan type $\gamma$ -

contraction

Fuzzy metric

\begin{abstract}
As other authors have been very interested in the topic of fixed points, we have obtained some results in this study that emphasize the importance of the fixed point theory. Kannan described a more general contraction than the Banach contraction that took its name and later Reich generalized this contraction further in metric spaces. In this paper, we have introduced some new contractions called Reich type $\gamma$-contraction and Kannan type $\gamma$-contraction which are generalization of $\gamma$-contraction and we have obtained some fixed point results for Reich type $\gamma$-contraction in non-Archimedean fuzzy metric spaces. We have presented a result about Kannan type-contraction. Furtermore, we have established an example about our main result.
\end{abstract}

\section{INTRODUCTION}

In 1922, with Banach's important introduction to the issue of fixed point theory, this issue became the focus of interest for the authors [1]. They generalized the Banach contraction theorem and produced new contractions, found new generalizations, and proved new theorems. There are many important studies in this area, and there are some contractions that we are inspired by. In 1968, Kannan defined a new contraction in metric spaces by generalizing the Banach contraction theorem [2]. Later, in 1971, Reich described a different and new contraction that is more general and has obtained new results regarding the fixed point theory issue [3].

On the other hand, Zadeh was the first to discover fuzzy metric space, which is one of the studied areas of fixed point theory [4]. The concept of a fuzzy metric space was introduced in different ways by some authors [5, 6]. Also, Istrăţescu [7] obtained a new form, based on the triangle inequality in the definition of fuzzy metric space. Later, the author defined a new space he obtained with this form as a nonArchimedean fuzzy metric space. Afterward, Banach type contraction theorem was proved and generalized in fuzzy metric spaces just like in metric spaces [8-22].

First of all, in this work we introduced a new contraction called Reich type $\gamma$-contraction and we prove a theorem regarding this contraction in non-Arcimedean fuzzy metric spaces. Next, we introduced a new contraction called Kannan type $\gamma$-contraction. We showed that these contractions we produce are a 
generalization of the $\gamma$-contraction done earlier. We proved some results and presented an example to show the validity of our theorem.

Now, we present some definitions we will use in our theorems.

Definition 1.1. [5] A binary operation $*:[0,1] \times[0,1] \rightarrow[0,1]$ is called a continuous triangular norm (in short, continuous $t-$ norm) if it satisfies the following conditions:

1. $*$ is commutative and associative,

2. $*$ is continuous,

3. $*(a, 1)=a$ for every $a \in[0,1]$,

4. $*(a, b) \leq *(c, d)$ whenever $a \leq c, b \leq d$ and $a, b, c, d \in[0,1]$.

Definition 1.2. [6] Let $\Omega$ is a nonempty set, * is a continuous t-norm and $M$ is a fuzzy set on $\Omega^{2} \times(0, \infty)$, satisfying the following conditions, for all $\varpi, v, \vartheta \in \Omega, s, t>0$ :

1. $M(\varpi, v, t)>0$,

2. $M(\varpi, v, t)=1$ if and only if $\varpi=v$,

3. $M(\varpi, v, t)=M(v, \varpi, t)$,

4. $M(\varpi, \vartheta, t+s) \geq M(\varpi, v, t) * M(v, \vartheta, s)$,

5. $M(\varpi, y, \cdot):(0, \infty) \rightarrow(0,1]$ is continuous.

If (NA) $M(\varpi, \vartheta, \max \{t, s\}) \geq M(\varpi, v, t) * M(v, \vartheta, s)$ is taken instead of the inequality (4), or equivalently,

$$
M(\varpi, \vartheta, t) \geq M(\varpi, v, t) * M(v, \vartheta, t)
$$

for all $\varpi, y, \vartheta \in \Omega, s, t>0$, then $(\Omega, M, *)$ is called a non-Archimedean fuzzy metric space [7].

Definition 1.3. Let $(\Omega, M, *)$ be a fuzzy metric space (or non-Arcimedean fuzzy metric space). Then

1. A sequence $\left\{\varpi_{\mathrm{n}}\right\}$ in $\Omega$ is said to converge to $\varpi$ in $\Omega$, denoted by $\varpi_{\mathrm{n}} \rightarrow \varpi$, if and only if $\lim _{n \rightarrow \infty} M\left(\varpi_{n}, \varpi, t\right)=1$ for all $t>0[11,16]$.

2. A sequence $\left\{\varpi_{n}\right\}$ is a Cauchy sequence if and only if for all $\varepsilon \in(0,1)$ and $t>0$, there exists $n_{0} \in \mathbb{N}$ such that $M\left(\varpi_{n}, \varpi_{m}, t\right)>1-\varepsilon$ for all $m, n \geq n_{0}[6,16]$.

3. If every Cauchy sequence is convergent, the fuzzy metric space (or the non-Archimedean fuzzy metric space) is said to be complete.

Definition 1.4. [22] Let $\gamma:[0,1) \rightarrow \mathbb{R}$ be a strictly increasing, continuous mapping and for each sequence $\left\{a_{n}\right\}_{n \in \mathbb{N}}$ of positive numbers $\lim _{n \rightarrow \infty} a_{n}=1$ if and only if $\lim _{n \rightarrow \infty} \gamma\left(a_{n}\right)=+\infty$. Let $\Gamma$ is the family of all $\gamma$ functions. A mapping $\mathrm{H}: \Omega \rightarrow \Omega$ is said to be a $\gamma$-contraction if there exists a $\delta \in(0,1)$ such that

$$
\mathrm{M}(\mathrm{H} \varpi, \mathrm{Hu}, \mathrm{s})<1 \Rightarrow \gamma(\mathrm{M}(\mathrm{H} \varpi, \mathrm{Tu}, \mathrm{s})) \geq \gamma(\mathrm{M}(\varpi, \mathrm{v}, \mathrm{s}))+\delta
$$

for all $\varpi, v \in X, s>0$ and $\gamma \in \Gamma$.

Example 1.5. Let $\gamma \in \Gamma$. Some examples for the $\gamma$ mapping are as follows:
1. $\frac{1}{1-\varpi}$
2. $\frac{1}{1-\varpi}+\varpi$ 

3. $\frac{1}{1-\varpi^{2}}$
4. $\frac{1}{\sqrt{1-\omega}}$

for all $\varpi \in[0,1)$.

\section{MAIN RESULTS}

Our aim in this section, is to present our new definitions and main result.

Definition 2.1. Let $H: \Omega \rightarrow \Omega$ be a mapping. If there exists a $\delta \in(0,1)$ and $\gamma \in \Gamma$ such that for all $\varpi, v \in$ $\Omega$ and $\mathrm{s}>0$ the following holds:

$$
\begin{aligned}
\mathrm{M}(\mathrm{H} \varpi, \mathrm{H} v, \mathrm{~s})<1 \Rightarrow & \\
& \gamma(\mathrm{M}(\mathrm{H} \varpi, \mathrm{H} v, \mathrm{~s})) \\
& \geq \gamma(\alpha \mathrm{M}(\varpi, v, \mathrm{~s})+\beta \mathrm{M}(\varpi, \mathrm{H} \varpi, \mathrm{s})+\zeta(\mathrm{M}(v, \mathrm{H} v, \mathrm{~s}))+\delta
\end{aligned}
$$

where $\alpha+\beta+\zeta=1$ and $\zeta \neq 1$, then $\mathrm{H}$ is called a Reich type $\gamma$-contraction.

Theorem 2.2. Let $\mathrm{H}: \Omega \rightarrow \Omega$ be a Reich type $\gamma$-contraction in a non-Archimedean fuzzy metric space. Then $\mathrm{H}$ has a unique fixed point.

Proof. Let $\varpi_{0} \in \Omega$ be arbitrary and fixed point. Define sequence $\left\{\varpi_{\mathrm{n}}\right\}$ by

$$
H \varpi_{n}=\varpi_{n+1} \text { for all } n \in \mathbb{N} \cup\{0\} .
$$

If $\varpi_{n}=\varpi_{n+1}$, then $\varpi_{n+1}$ is a fixed point of $H$ then the result is proved. Let $M_{n}=M\left(\varpi_{n}, \varpi_{n+1}, s\right)$ for all $n \in \mathbb{N} \cup\{0\}$. We suppose that $\varpi_{n} \neq \varpi_{n+1}$ for all $n \in \mathbb{N} \cup\{0\}$. Therefore by inequality (1), we have

$$
\begin{aligned}
& \gamma\left(M_{n}\right) \\
& =\gamma\left(M\left(\varpi_{n}, \varpi_{n+1}, s\right)\right)=\gamma\left(M\left(H \varpi_{n-1}, H \varpi_{n}, s\right)\right) \\
& \geq \gamma\left(\alpha M\left(\varpi_{n-1}, \varpi_{n}, s\right)+\beta M\left(\varpi_{n-1}, H \varpi_{n-1}, s\right)+\zeta M\left(\varpi_{n}, H \varpi_{n}, s\right)\right)+\delta \\
& =\gamma\left(\alpha M\left(\varpi_{n-1}, \varpi_{n}, s\right)+\beta M\left(\varpi_{n-1}, \varpi_{n}, s\right)+\zeta M\left(\varpi_{n}, \varpi_{n+1}, s\right)\right)+\delta \\
& =\gamma\left((\alpha+\beta) M_{n-1}+\zeta M_{n}\right)+\delta .
\end{aligned}
$$

Since $\gamma$ is a strictly increasing, we have

$$
M_{n}>(\alpha+\beta) M_{n-1}+\zeta M_{n}
$$

and hence

$$
(1-\zeta) M_{n}>(\alpha+\beta) M_{n-1}
$$

for all $\mathrm{n} \in \mathbb{N}$. Since $\alpha+\beta+\zeta=1$ and $\zeta \neq 1$, we get

for all $\mathrm{n} \in \mathbb{N}$. Then, we obtain

$$
M_{n}>M_{n-1}
$$

$$
\gamma\left(M_{n}\right) \geq \gamma\left(M_{n-1}\right)+\delta .
$$

By repeating the above process, we get

$$
\gamma\left(\mathrm{M}_{\mathrm{n}}\right) \geq \gamma\left(\mathrm{M}_{\mathrm{n}-1}\right)+\delta \geq \gamma\left(\mathrm{M}_{0}\right)+\mathrm{n} \delta
$$


for all $n \in \mathbb{N}$. Letting $n \rightarrow \infty$, from the inequality (3) we have

$$
\lim _{n \rightarrow \infty} \gamma\left(M_{n}\right)=+\infty
$$

Then, we have

$$
\lim _{n \rightarrow \infty} M_{n}=\lim _{n \rightarrow \infty} M\left(\varpi_{n}, \varpi_{n+1}, s\right)=1 .
$$

Now, we assume that $\left\{\varpi_{n}\right\}$ is not a Cauchy sequence. Then, there are $\varepsilon \in(0,1)$ and $s_{0}>0$ such that for all $p \in \mathbb{N}$ there exist $n(p), m(p) \in \mathbb{N}$ with $m(p)>n(p) \geq p$ and

$$
\mathrm{M}\left(\varpi_{\mathrm{n}(\mathrm{p})}, \varpi_{\mathrm{m}(\mathrm{p})}, \mathrm{s}_{0}\right) \leq 1-\varepsilon .
$$

Assume that $m(p)$ is the least integer exceeding $n(p)$ satisfying the inequality (5). Equivalently

$$
M\left(\varpi_{m(p)-1}, \varpi_{n(p)}, s_{0}\right)>1-\varepsilon
$$

and so, for all $p \in \mathbb{N}$, we get

$$
\begin{aligned}
& 1-\varepsilon \geq M\left(\varpi_{n(p)}, \varpi_{m(p)}, s_{0}\right) \\
& \geq M\left(\varpi_{m(p)-1}, \varpi_{m(p)}, s_{0}\right) * M\left(\varpi_{m(p)-1}, \varpi_{n(p)}, s_{0}\right) \\
& \geq M\left(\varpi_{m(p)-1}, \varpi_{m(p)}, s_{0}\right) *(1-\varepsilon) .
\end{aligned}
$$

By taking limit as $\mathrm{p} \rightarrow \infty$ in (7) and using (4), we deduce that

From (NA), we get

$$
\lim _{\mathrm{p} \rightarrow \infty} \mathrm{M}\left(\varpi_{\mathrm{n}(\mathrm{p})}, \varpi_{\mathrm{m}(\mathrm{p})}, \mathrm{s}_{0}\right)=1-\varepsilon
$$

$$
\begin{aligned}
M\left(\varpi_{m(p)+1}, \varpi_{n(p)+1}, s_{0}\right) \geq M\left(\varpi_{m(p)+1}, \varpi_{m(p)}, s_{0}\right) & * M\left(\varpi_{m(p)}, \varpi_{n(p)}, s_{0}\right) \\
& * M\left(\varpi_{n(p)}, \varpi_{n(p)+1}, s_{0}\right) .
\end{aligned}
$$

Letting $p \rightarrow \infty$ in (9), we get

$$
\lim _{\mathrm{p} \rightarrow \infty} \mathrm{M}\left(\varpi_{\mathrm{n}}(\mathrm{p})+1, \varpi_{m(p)+1}, \mathrm{~s}_{0}\right) \geq 1-\varepsilon .
$$

By applying the inequality (1) with $\varpi=\varpi_{\mathrm{m}(\mathrm{p})}$ and $v=\varpi_{\mathrm{n}(\mathrm{p})}$

$$
\begin{aligned}
& \gamma\left(M\left(\varpi_{n(p)+1}, \varpi_{m(p)+1}, s_{0}\right)\right) \\
& \geq \gamma\left(\alpha M\left(\varpi_{n(p)}, \varpi_{m(p)}, s_{0}\right)+\beta M\left(\varpi_{n(p)}, \varpi_{n(p)+1}, s_{0}\right)\right. \\
& \left.\quad+\zeta M\left(\varpi_{m(p)}, \varpi_{m(p)+1}, s_{0}\right)\right)+\delta .
\end{aligned}
$$

Taking the limit as $p \rightarrow \infty$ in (11), by using the continuity of $\gamma$, applying (1), from (8), (10), we obtain

$$
\gamma(1-\varepsilon) \geq \gamma(\alpha(1-\varepsilon)+\beta+\zeta)+\delta .
$$

Since $\gamma$ is a strictly increasing, we have

Since $\alpha+\beta+\zeta=1$ and $\zeta \neq 1$, we get

$$
(1-\alpha)(1-\varepsilon)>\beta+\zeta
$$

$$
1-\varepsilon>1
$$


This is a contradiction. Thus $\left\{\varpi_{\mathrm{n}}\right\}$ is a Cauchy sequence. Since $\Omega$ is complete, there exists $\mathrm{z} \in \Omega$ such that

$$
\lim _{\mathrm{n} \rightarrow \infty} \varpi_{\mathrm{n}}=\mathrm{z}
$$

Now we need to show that the point $\mathrm{z}$ is a fixed point of $\mathrm{H}$. Suppose the contrary, that is $\mathrm{z} \neq \mathrm{Hz}$. So, there exists $n_{0} \in \mathbb{N}$ and a subsequence $\left\{\varpi_{n_{p}}\right\}$ of $\left\{\varpi_{n}\right\}$ such that $M\left(H \varpi_{n_{p}}, H z, s\right)<1$ for all $n_{p} \geq n_{0}$. It follows from (1), property of $\gamma$,

$$
\begin{aligned}
\gamma\left(\mathrm{M}\left(\varpi_{\mathrm{n}_{\mathrm{p}}+1}, \mathrm{~Hz}, \mathrm{~s}\right)\right) & =\gamma\left(\mathrm{M}\left(\mathrm{H} \varpi_{\mathrm{n}_{\mathrm{p}}}, \mathrm{Hz}, \mathrm{s}\right)\right) \\
& \geq \gamma\left(\alpha \mathrm{M}\left(\varpi_{\mathrm{n}_{\mathrm{p}}}, \mathrm{z}, \mathrm{s}\right)+\beta \mathrm{M}\left(\varpi_{\mathrm{n}_{\mathrm{p}}}, \mathrm{H} \varpi_{\mathrm{n}_{\mathrm{p}}}, \mathrm{s}\right)+\zeta \mathrm{M}(\mathrm{z}, \mathrm{Hz}, \mathrm{s})\right)+\delta \\
& =\gamma\left(\alpha \mathrm{M}\left(\varpi_{\mathrm{n}_{\mathrm{p}}} \mathrm{z}, \mathrm{s}\right)+\beta \mathrm{M}\left(\varpi_{\mathrm{n}_{\mathrm{p}}}, \varpi_{\mathrm{n}_{\mathrm{p}}+1}, \mathrm{~s}\right)+\zeta \mathrm{M}(\mathrm{z}, \mathrm{Hz}, \mathrm{s})\right)+\delta
\end{aligned}
$$

Since property of $\gamma$, we obtain

$$
M\left(\varpi_{n_{p}+1}, H z, s\right)>\alpha M\left(\varpi_{n_{p}}, z, s\right)+\beta M\left(\varpi_{n_{p}}, \varpi_{n_{p}+1}, s\right)+\zeta M(z, H z, s) .
$$

for all $n \in \mathbb{N}$. Letting $n \rightarrow \infty$ in (12), we have

$$
(1-\zeta) \mathrm{M}(\mathrm{z}, \mathrm{Hz}, \mathrm{s})>\alpha+\beta .
$$

Since $\alpha+\beta+\zeta=1$ and $\zeta \neq 1$, we get

$$
\mathrm{M}(\mathrm{z}, \mathrm{Hz}, \mathrm{s})>1
$$

which is a contradiction. Hence, $\mathrm{z}=\mathrm{Hz}$. Uniqueness: Let $\mathrm{z}_{1}, \mathrm{z}_{2}$ be two fixed points of $\mathrm{H}$. Suppose that $\mathrm{z}_{1} \neq \mathrm{z}_{2}$, then we get $\mathrm{Hz}_{1} \neq \mathrm{Hz}_{2}$. From (1), we have

$$
\begin{aligned}
\gamma\left(\mathrm{M}\left(\mathrm{z}_{1}, \mathrm{z}_{2}, \mathrm{~s}\right)\right) & =\gamma\left(\mathrm{M}\left(\mathrm{Hz}_{1}, \mathrm{~Hz}_{2}, \mathrm{~s}\right)\right) \\
\geq \gamma\left(\alpha \mathrm{M}\left(\mathrm{z}_{1}, \mathrm{z}_{2}, \mathrm{~s}\right)+\beta \mathrm{M}\left(\mathrm{z}_{1}, H \mathrm{z}_{1}, \mathrm{~s}\right)+\zeta \mathrm{M}\left(\mathrm{z}_{2}, H \mathrm{H}_{2}, \mathrm{~s}\right)\right)+\delta & =\gamma\left(\alpha \mathrm{M}\left(\mathrm{z}_{1}, \mathrm{z}_{2}, \mathrm{~s}\right)+\beta \mathrm{M}\left(\mathrm{z}_{1}, \mathrm{z}_{1}, \mathrm{~s}\right)+\zeta \mathrm{M}\left(\mathrm{z}_{2}, \mathrm{z}_{2}, \mathrm{~s}\right)\right)+\delta \\
& =\gamma\left(\alpha \mathrm{M}\left(\mathrm{z}_{1}, \mathrm{z}_{2}, \mathrm{~s}\right)+\beta+\zeta\right)+\delta .
\end{aligned}
$$

Since $\gamma$ is a strictly increasing, we have

$$
\mathrm{M}\left(\mathrm{z}_{1}, \mathrm{z}_{2}, \mathrm{~s}\right)>\alpha \mathrm{M}\left(\mathrm{z}_{1}, \mathrm{z}_{2}, \mathrm{~s}\right)+\beta+\zeta
$$

Then, we have

Since $\alpha+\beta+\zeta=1$ and $\zeta \neq 1$, we get

$$
(1-\alpha) M\left(z_{1}, z_{2}, s\right)>\beta+\zeta
$$

$$
\mathrm{M}\left(\mathrm{z}_{1}, \mathrm{z}_{2}, \mathrm{~s}\right)>1
$$

That is a contradiction. Hence, $\mathrm{M}\left(\mathrm{z}_{1}, \mathrm{z}_{2}, \mathrm{~s}\right)=1$, that is, $\mathrm{z}_{1}=\mathrm{z}_{2}$.

Corollary 2.3. Let $\mathrm{H}: \Omega \rightarrow \Omega$ be $\gamma$-contraction in a non-Archimedean fuzzy metric space. Then $\mathrm{H}$ has a unique fixed point.

Proof. If we take $\beta, \zeta=0$ in the proof of Theorem 2.2, the proof of Corollary 2.3 is completed.

Remark 2.4. Corollary 2.3 is an result in the paper [17]. That is, Theorem 2.2 is a generalization of Corollary 2.3. 
Definition 2.5. Let $H: \Omega \rightarrow \Omega$ be a mapping. If there exists a $\delta \in(0,1)$ and $\gamma \in \Gamma$ such that for all $\varpi, v \in$ $\Omega$ and $\mathrm{s}>0$ the following holds:

$$
\mathrm{M}(\mathrm{H} \varpi, \mathrm{Hv}, \mathrm{s})<1 \Rightarrow \gamma(\mathrm{M}(\mathrm{H} \varpi, \mathrm{Hv}, \mathrm{s})) \geq \gamma(\beta \mathrm{M}(\varpi, \mathrm{H} \varpi, \mathrm{s})+\zeta(\mathrm{M}(\mathrm{v}, \mathrm{Hv}, \mathrm{s}))+\delta
$$

where $\beta+\zeta=1$ and $\zeta \neq 1$, then $H$ is called a Kannan type $\gamma$-contraction.

Corollary 2.6. Let $\mathrm{H}: \Omega \rightarrow \Omega$ be a Kannan type $\gamma$-contraction in a non-Archimedean fuzzy metric space. Then $\mathrm{H}$ has a unique fixed point.

Proof. By taking $\alpha=0$ in Theorem 2.2, the proof of Theorem 2.2 is complete.

Example 2.7. Let $\Omega=\Omega_{1} \cup \Omega_{2}$ where $\Omega_{1}=\{1,2,3\}, \Omega_{2}=[4,6]$ and $\mathrm{a} * \mathrm{~b}=\min \{\mathrm{a}, \mathrm{b}\}$,

$$
M(\varpi, v, s)= \begin{cases}1 /(1+\max \{\varpi, v\}), & \varpi \neq v, \\ 1 & \varpi=v,\end{cases}
$$

for all s $>0$. Consider $\gamma:[0,1) \rightarrow \mathbb{R}$ such that $\gamma(\varpi)=\frac{1}{\sqrt{1-\varpi^{2}}}$ for all $\left.\varpi \in 0,1\right)$ and define $H: \Omega \rightarrow \Omega$ by

$$
H(\varpi)= \begin{cases}1, & \varpi \in \Omega_{1} \\ 2, & \varpi \in \Omega_{2}\end{cases}
$$

Now, we have to show that $H$ is a Reich type $\gamma$-contraction. Let $\mathrm{M}(\mathrm{H} \varpi, \mathrm{Hv}, \mathrm{s})<1$, so for all $\varpi \in \Omega_{1}$ and $v \in \Omega_{2}$, we have the following equations:

$$
\begin{aligned}
& \mathrm{M}(\mathrm{H} \varpi, \mathrm{Hv}, \mathrm{s})=\frac{1}{1+\max \{\mathrm{H} \varpi, \mathrm{H} v\}}=\frac{1}{1+2}=\frac{1}{3}, \\
& \mathrm{M}(\varpi, \mathrm{v}, \mathrm{s})=\frac{1}{1+\max \{\varpi, v\}}=\frac{1}{1+v^{\prime}} \\
& \mathrm{M}(\varpi, \mathrm{H} \varpi, \mathrm{s})=\frac{1}{1+\max \{\varpi, \mathrm{H} \varpi\}}=\frac{1}{1+\varpi} \\
& \mathrm{M}(\mathrm{v}, \mathrm{Hv}, \mathrm{s})=\frac{1}{1+\max \{v, \mathrm{Hv}\}}=\frac{1}{1+v} .
\end{aligned}
$$

Now we must show that the inequality (1) is satisfied

$$
\gamma(M(H \varpi, H v, s)) \geq \gamma(\alpha M(\varpi, v, s)+\beta M(\varpi, H \varpi, s)+\zeta(M(v, H v, s))+\delta
$$

is equivalent to

$$
M(H \varpi, H v, s))>\alpha M(\varpi, v, s)+\beta M(\varpi, H \varpi, s)+\zeta(M(v, H v, s)) .
$$

By choosing $\alpha=\frac{2}{8}, \beta=\frac{5}{8}$ and $\zeta=\frac{1}{8}$, we have

$$
\frac{1}{3}>\alpha \frac{1}{1+v}+\beta \frac{1}{1+\varpi}+\zeta \frac{1}{1+v}
$$

is equivalent to

$$
\frac{1}{3}>(\alpha+\zeta) \frac{1}{1+v}+\beta \frac{1}{1+\varpi}
$$

For $\min \{\varpi\}=1$ and $\min \{u\}=4$, we get

$$
\frac{1}{3}>\frac{7}{8} \frac{1}{5}+\frac{1}{8} \frac{1}{2}=\frac{19}{80} .
$$


So, there exists a $\delta \in(0,1)$ such that for all $\varpi \in \Omega_{1}$ and $v \in \Omega_{2}$ the inequality (1) is satisfied. Hence, all the conditions of Theorem 2.2 hold, that is, $\varpi=1$ is the unique fixed point of $\mathrm{H}$.

\section{CONFLICTS OF INTEREST}

No conflict of interest was declared by the author.

\section{REFERENCES}

[1] Banach, S., "Sur les opérations dans les ensembles abstraits et leurs applications aux équations intégrales", Fundamenta Mathematicae, 3: 133-181, (1922).

[2] Kannan, R., "Some results on fixed points", Bulletin of Calcutta Mathematical Society, 60: 7176, (1968).

[3] Reich, R., "Some remarks concernin contraction mappings", Canadian Mathematical Bulletin, 14: 121-124, (1971).

[4] Deng, Z., "Fuzzy pseudometric spaces", Journal of Mathematical Analysis and Applications, 86: 74-95, (1922).

[5] Salimi, P., Vetro, C., and Vetro, P., "Some new fixed point results in non-Archimedean fuzzy metric spaces", Nonlinear Analysis: Modelling and Control, 18(3): 344-358, (2013).

[6] George, A., and Veeramani P., "On some results in fuzzy metric spaces", Fuzzy Sets and Systems, 64, 395-399, (1994).

[7] Istrăţescu, V., "An introduction to theory of probabilistic metric spaces with applications”, Ed, Tehnică, Bucureşti, in Romanian, (1974).

[8] Grabiec, M., "Fixed points in fuzzy metric spaces", Fuzzy Sets and Systems, 27: 385-389, (1988).

[9] Gregori, V., and Sapena, A., "On fixed-point theorems in fuzzy metric spaces", Fuzzy Sets Systems, 125: 245-252, (2002).

[10] Vetro, C., and Vetro, P., "Common fixed points for discontinuous mappings in fuzzy metric spaces", Rendiconti del Circolo Matematico di Palermo, 57: 295-303, (2008).

[11] Kramosil, I., and Michalek, J., "Fuzzy metric and statistical metric spaces", Kybernetika, 11: 336-344, (1975).

[12] Altun, I., and Mihet, D., "Ordered non-Archimedean fuzzy metric spaces and some fixed point results", Fixed Point Theory and Applications, 2010: (2010).

[13] Hussain, N., Hezarjaribi M., and Salimi, P., "Suzuki type theorems in triangular and nonArchimedean fuzzy metric spaces with application", Fixed Point Theory and Applications, 2015: 134, (2015).

[14] Cho, Y. J., "Fixed points in fuzzy metric spaces", Journal of Fuzzy Mathematics, 5(4): 949-962, (1997). 
[15] Schweizer, B., and Sklar, A., "Statistical metric spaces", Pacific Journal of Mathematics, 10: 385-389, (1960).

[16] Schweizer, B., and Sklar, A., "Probabilistic Metric Spaces", North-Holland, Amsterdam, (1983).

[17] Özgür, N. Y., and Taş, N., "Some fixed-circle theorems on metric spaces", Bulletin of the Malaysian Mathematical Sciences Society, 42: 1433-1449, (2019).

[18] Taş, N., "Bilateral type solutions to the fixed-circle problem with recti-ed linear units application", Turkish Journal of Mathematics, 44: 1330- 1344, (2020).

[19] Aydi, H., Taş, N., Özgür, N. Y., and Mlaiki, N., "Fixed-discs in rectangular metric spaces", Symmetry, 11(2): 294, (2019).

[20] Sahin, H., "Best proximity point theory on vector metric spaces", Communications Faculty of Sciences University of Ankara Series A1 Mathematics and Statistics, 70 (1): 130-142, (2021).

[21] Altun, I., Sahin, H., and Turkoglu, D., "Caristi-Type fixed point theorems and some generalizations on M-metric space", Bulletin of the Malaysian Mathematical Sciences Society, 43: 2647-2657, (2020).

[22] Sezen, M. S., "Fixed point theorems for new type contractive mappings", Journal of Function Spaces, Article ID: 2153563, (2019). 